\title{
The impact of asynchronous trading on Epps effect. \\ Comparative study on Warsaw Stock Exchange and Vienna Stock Exchange ${ }^{1}$
}

\section{Introduction}

A very important research topic is describing the way in which different asset price movements are correlated. Modern portfolio theory methods are based on observed correlations between returns at daily or larger time scales. One expects that coarse scale correlations originate from intraday movements that are strongly correlated. This implies the important question of how to obtain better estimates of such correlations by using high-frequency data. Here, one can observe an analogous paradox as for volatility estimation under microstructure noise. It is surprising that the correlation coefficient is an increasing function of the time resolution, and that correlation very quickly decays and almost vanishes at a very high frequency. The dependence of correlations between stock prices on the sampling frequency of time series involves a phenomenon called the Epps effect. The actual correlations between returns of stocks decrease as the sampling frequency of data increases. The Epps effect has received considerable attention, not only from economists but also from mathematicians and theoretical physicists. But there are only a few contributions to the subject.

The Epps effect seems to be an unexpected phenomenon at a first glance. However it is simply explainable by different factors; e.g., the asynchrony of trade times. It is clear that 1-minute returns are, in fact, returns for the last trade prices

* AGH University of Science and Technology in Krakow, Faculty of Management, Department of Applications of Mathematics in Economics; e-mails: henryk.gurgul@gmail.com; artur.machno@gmail.com

${ }^{1}$ Financial support for this paper comes from the National Science Center of Poland (Research Grant DEC-2012/05/B/HS4/00810) and is gratefully acknowledged by Henryk Gurgul.

Financial support for this paper comes from the Dean of Faculty of Management, AGH University (statutory activity no. 15/11.200.296) and is gratefully acknowledged by Artur Machno. 
before the appropriate times. If one takes very short horizons or non-liquid assets, then some returns are calculated for the same trade. Therefore, those returns are, of course, zero. They do not give any additional contribution to correlations. It is clear that the situation where there are no trades in a time interval and, therefore, no correlation is the extreme case. However, even if the asynchrony is not in its extreme case, the Epps effect is present.

Financial transactions supply information about current prices. In addition, they assure fixing of the market value of traded stocks until the next trade takes place. From this point of view, the financial literature considers two main groups of contributions to the Epps effect. The first group of authors is concerned with the fact that price dynamics are not synchronous across stocks. This means that transactions are conducted at different times for different shares. The second one relates the Epps effect to actual lagged correlations. The main reason for this phenomenon is the temporary or permanent effect of individual trades on the price dynamics.

The main goal of this paper is to compare the Epps effect on the stock markets in Vienna and Warsaw. There are indications suggesting that the VSE and WSE may be linked. The linkage is mostly indirect because of a strong relationship with Germany. First of all, the VSE and WSE are somewhat similar in some aspects. Both stock markets are of similar size. The main indices of these markets are among the largest in Central and Eastern Europe, and they have been quoted for a similar period of time ${ }^{2}$. In addition, the VSE and WSE have been competing markets in recent years. On the other hand, the VSE is a developed market, while the WSE still belongs to emerging markets despite of development in recent years. The important channel of interrelations may be foreign trade. Germany is the most important trading partner for both the Austrian and Polish economies.

The computations in the empirical part of this paper are made in the R environment. All codes that have been used are available from the authors upon request. Additionally, the authors can provide the data and codes needed to reproduce all tables and plots used in this article.

The main finding of the study is that the Epps effect is surprisingly different for Austrian and Polish assets. The analysis shows that all considered Polish pairs exhibit a similar relationship in terms of correlation and the impact of the asynchrony on it. Moreover, the results for the WSE are as generally expected. In the case of Austrian pairs, we have found several aberrations. Only one analyzed Austrian pair has similar properties to all Polish pairs.

\footnotetext{
${ }^{2}$ ATX20 index (VSE) is quoted from January 2 1991, and the WIG20 index (WSE) is published from
} April 16, 1994. 
To the best of our knowledge, this is the first contribution dealing simultaneously with the Epps effect on the stock markets in Vienna and Warsaw. The comparison of the Epps effect (and particularly the asynchrony trading impact in it) on stocks from the given stock markets is of the most valuable and novel part of the paper.

The content of this paper is as follows; the next section briefly reviews the most important recent contributions concerning the Epps effect, and the main conjectures are presented in the third section. The following section presents the methodology, and the fifth section is concerned with the dataset and empirical results. Finally, we summarize the major conclusions and suggest directions for future research.

\section{Literature review}

In his contribution in 1979, Thomas Wake Epps provides empirical evidence of a dramatic drop in correlations between stocks with a decreasing sampling time horizon. This observation has been reported for different markets.

In the opinion of most of the authors, it is not obvious which type of price formation process is the source of the Epps effect. Lundin et al. (1998) found an essential inverse link between correlation and activity. They found that growth in the quantity of assets traded reduces the Epps effect. This observation is in favor of the view that non-synchronicity is the main source of correlation drop at higher frequencies.

According to several authors (e.g., Conlon et al., 2009), a number of financial mechanisms of stock price and trading-volume formation are the source of the Epps effect. The goal of Mastromatteo et al. (2011) was not to derive a complete description of the Epps effect. The contributors stressed that they rather aimed at identifying statistical causes that can be compensated for directly, without the need to adjust parameters, model calibrations, or an optimal sampling frequency. They identified two major causes: the asynchrony of the time series and the impact of discretization by tick-size.

Toth and Kertesz (2009) assumed that there is a price of an asset at any time. However, it is observable only at the times of trade. This assumption is suggested by the observed Epps effect. In the opinion of the authors, the impact of the asynchrony is weak in relation to the impact of a static lag for which they developed a model. The authors think that the source of decline of the Epps effect with time is increased market efficiency.

Munnix et al. (2010) found that the calculated correlation consists of an actual correlation (the coefficient that would be observed if prices were quoted 
continuously and priced with a continuous value) and an uncorrelated part caused by asynchronous trading. The authors demonstrated that the asynchrony of trades and the decimalization of stock prices are the main sources of decline of the correlation coefficients towards smaller return intervals (i.e., the Epps effect). Munnix et al. (2010) argued that these distortions are of a purely statistical origin and depend on the properties of the time series.

The most recent contribution by Gurgul and Machno (2015) is concerned with the impact of asynchronous trading on the Epps effect for some stocks quoted on the WSE in both theoretical and empirical aspects.

To conclude, there are certainly many phenomena contributing to the Epps effect. However, one of the most important sources of the Epps effect seems to be the asynchronous trading.

To the best of the knowledge of the authors, this is the one of the first studies concerning the impact of asynchronous trading on the Epps effect in the stock exchanges in Vienna and Warsaw.

\section{Description of the dataset}

In this part of the paper, we briefly describe the stocks chosen for the analysis. We use four stocks for both stock exchanges under study. Each four pairs are the most-frequently-traded stocks of a particular exchange. Below, we give a short data description of datasets from both stock exchanges. Our analysis is based on the tick-by-tick data from both stock exchanges. We use the most recent tick-bytick data available to us for the Vienna and Warsaw stock exchanges (2013 and 2014, respectively).

\subsection{Warsaw Stock Exchange data}

The dataset from the Warsaw Stock Exchange used in our contribution encompass every operation that took place on the WSE from 1.01.2014 to September 22.2014. We stress that the electronic system of the WSE has been changed since August 01.2013. The most important change from the point of view of our analysis is the rise of exactness of the trade time from seconds to microseconds. In this way, it overcame the problem of two or more transactions at the same time. Since this date, each two transactions are distinguishable in the time. The trade takes place on the WSE between 9:00 a.m. and 5:05 p.m. In addition, there is a break from 4:50 p.m. to 5:00 p.m. Moreover, during the last five minutes, trade on the WSE is not conducted as it is during normal 
hours. To avoid biases in the results caused by data, we restrict our dataset and take into account the transactions conducted not later than 4:50 p.m. in our analysis. After these restrictions, the time span of our analysis includes $\mathbf{4 7 0}$ minutes of trade each day.

The WSE is the biggest stock exchange from the countries that entered the European Union in 2004 or since. The capitalization of the WSE is about 1.2 trillion PLN. Now, 487 stocks are listed in the Warsaw Stock Exchange.

The transactions conducted on the WSE are quoted in Polish zloty (PLN). The exchange rates during the first days of February 2016 are approximately given by $1 \mathrm{USD}=4.04 \mathrm{PLN}$ and $1 \mathrm{EUR}=4.41 \mathrm{PLN}$. In the text, all prices are expressed in PLN for Polish stocks.

The most-frequently-traded stocks on the WSE during the period under study (January 01, 2014 - September 22, 2014) were the four stocks described below.

KGHM Polska Miedź (KGHM) is the largest mining and metallurgy company in Poland. Its main product is copper. The market value of the company is approximately 19 billion PLN. The company employs about 30,000 employees.

Powszechna Kasa Oszczędności Bank Polski (PKOBP) is the largest Polish bank. The market value of the bank is about 36 billion PLN. PKOBP employs about 26,000 employees.

Polska Grupa Energetyczna (PGE) is the largest Polish energy producer and provider. The biggest investor of PGE is the Polish government (it owns more than $60 \%$ of the shares of PGE). The value of the company is about 33 billion PLN. The company employs about 41,000 employees.

Powszechny Zakład Ubezpieczeń (PZU) is the largest Polish financial institution. Its business area is insurance, and it dominates the Polish insurance market. The value of the company is about 37 billion PLN. The company employs more than 17,000 employees.

\subsection{Vienna Stock Exchange data}

The dataset from the Vienna Stock Exchange used in this contribution includes every operation that took place on the VSE from January 01, 2013 to December 31,2013 . The exactness of the trade time for this dataset is 0.01 seconds. The continuous trade hours on the VSE are during the time span, from 9:00 a.m. to $12: 00$ p.m. and then 12:03 p.m. to 5:30 p.m. This time span consists of 507 minutes of trade each day, with a 3-minute gap in the middle.

The VSE is a relatively small mature market. The capitalization of the VSE is about 87.7 billion EUR. There are 96 listed companies, and the main equity index is the ATX20 (consisting of 20 companies). 
All transactions on the VSE are quoted in Euro (EUR). In the text, we present all values in EUR for Austrian stocks.

The most-frequently-traded assets on the VSE during the period January 01, 2013 to December 31, 2013 are described below.

Erste Group Bank AG (EBS) is one of the biggest financial institutions in Central and Eastern Europe. The main subsidiaries of the group are Austrian Erste Bank der oesterreichischen Sparkassen AG, Czech Česká spořitelna, Slovakian Slovenská sporitel’ňa, Hungarian Erste Bank Hungary, Croatia Erste \& Steiermärkische Bank, Serbian Erste Bank a.d. Novi Sad, Romanian Banca Comercială Română, and Slovenian Banka Sparkasse. The value of the group is about 10 billion EUR, and it employs about 46,000 employees.

OMV (formerly Österreichische Mineralölverwaltung) is an international oil and gas company. It is active in many areas of the oil and gas field, like exploration, production, refining, etc. The company employs about 26,000 employees.

Andritz AG (ANDR) is a plant engineering company. It consists of Andritz Hydro, Andritz Pulp and Paper, Andritz Metals, Andritz Separation, and Andritz Feed \& Biofuel. The value of the company is about 1 billion EUR, and it employs about 24,000 employees.

Voestalpine AG (VOE) is an international steel-based corporation. Among others, the company is active in railway systems and the tool steel industry. The value of the company is about 5 billion EUR, and it employs about 46,000 employees.

\section{Duration}

In this part of the paper, visualization of the data is presented. We have randomly chosen two days (namely, February 19 and July 7, 2014) for the descriptive analysis for the WSE. Figure 1 presents all trades of the four analyzed stocks from the WSE on the examplary day. The stocks are very liquid; therefore, it is hard to see differences in the durations. By duration, we mean the time that elapses between trades.

Analogously, we have randomly chosen two days (namely, April 5 and May 15, 2013) for the descriptive analysis for the VSE. Figure 2 presents all trades of the four analyzed stocks from the VSE on the exemplary day.

In Table 1, the numbers of trades of the stocks on the VSE and WSE during the analyzed periods are collected. We also included summary statistics for the duration on both stock exchanges. The duration distribution in the case of the VSE and WSE that are strongly linked with greater markets seems to be an interesting topic; however, the modeling of duration is not the topic of this paper. 


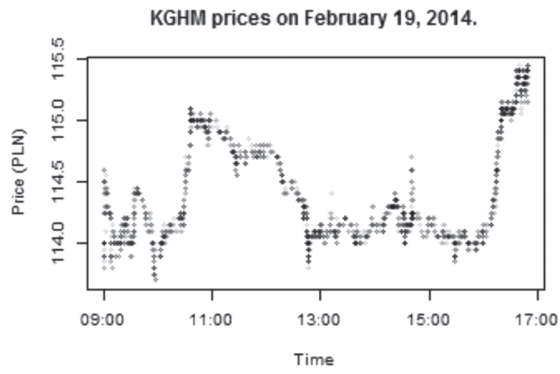

PKO prices on February 19, 2014.

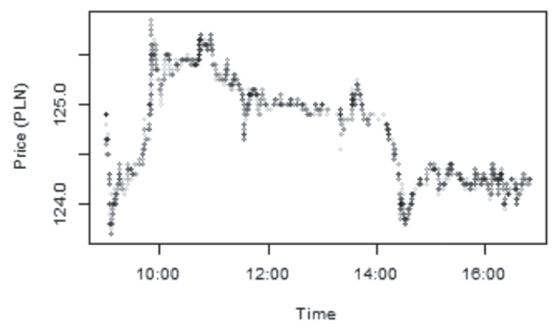

PGE prices on February 19, 2014.

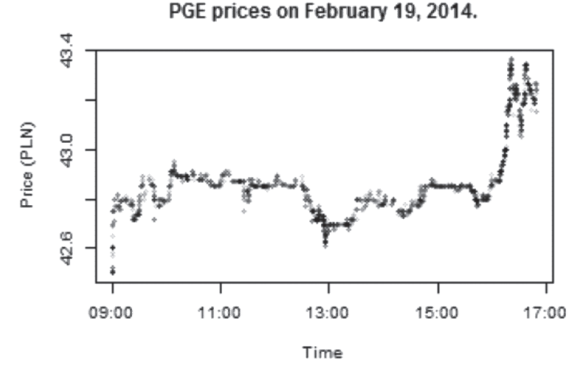

PZU prices on February 19, 2014.

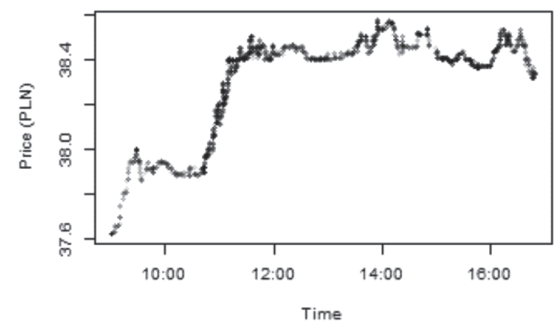

Figure 1. All transactions of KGHM, PKO, PGE, and PZU on February 19, 2014.

The color of dots is grey with $90 \%$ transparency

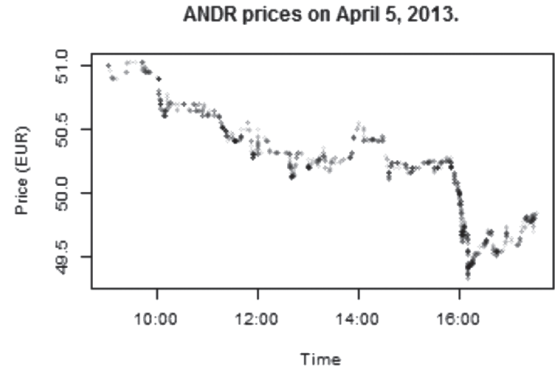

EBS prices on April 5, 2013.

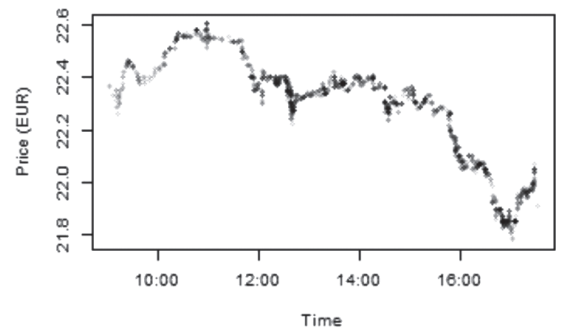

OMV prices on April 5, 2013.

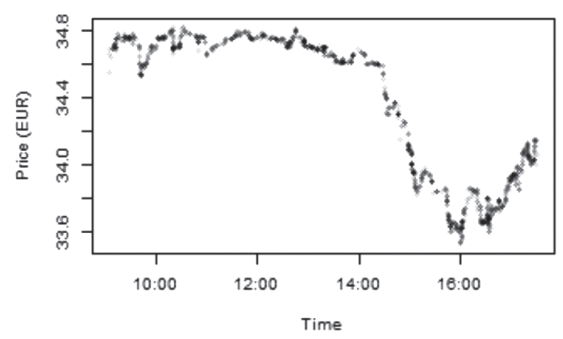

VOE prices on April 5, 2013.

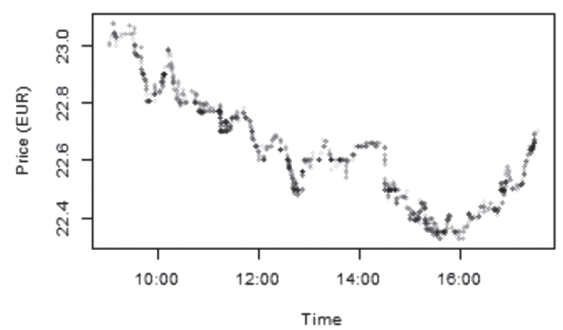

Figure 2. All transactions of ANDR, EBS, OMV, and VOE on April 5, 2014.

The color of dots is grey with $90 \%$ transparency 
The descriptive statistics are quite similar for the stocks under study and days on the WSE. One can see that the minimums are the same ( 1 microsecond). This is probably caused by the electronic system. The first quartiles take the values from the interval between 4 and 7 microseconds. This is a very small interval. Here raises the question: How is it possible that a quarter of operations take place immediately after the preceding one? Means and the number of transactions are strictly related characteristics of trade on a stock exchange. The mean is the total trade time on the day ( 470 minutes, 28,200 seconds) denominated by the number of transactions. The third quartiles start from 4.1 seconds, and their upper bound is 5.9 seconds. This also is not a wide interval. The wider intervals determine maximums. They vary from 14 to 26.3 minutes. Contrary to other quartiles, the median of the duration quite strongly varies for the analyzed datasets. This observation is even strongly pronounced if the analysis is conducted separately for various days. The distribution of durations is "U" shaped; this means that the durations are either very low or relatively large. There are only a few little durations near the median. This is probably the reason for the strong variation of the median across stocks and days.

The exactness of the data from the VSE due to its electronic system is lower; therefore, the minimum is zero for every stock. The other statistics show that the trading is much more intense on the WSE as compared to the VSE. What is apparent and worth further, deeper analysis is that the first quartiles for Polish stocks are more than 10,000 times lower than Austrian stocks, while means and median are about 10 times lower. It might be the case that the electronic systems work essentially differently on those stock exchanges.

Table 1

Descriptive statistics for tick data

\begin{tabular}{|c|c|c|c|c|c|c|c|}
\hline & Trades & Min. & $\mathbf{Q}_{1}$ & Median & Mean & $\mathbf{Q}_{3}$ & Max. \\
\hline KGHM & 695202 & 0.000001 & 0.000004 & 0.0522 & 7.381 & 4.3810 & 844 \\
\hline PКОBP & 645032 & 0.000001 & 0.000005 & 0.0911 & 7.955 & 4.1330 & 977 \\
\hline PGE & 497428 & 0.000001 & 0.000005 & 0.0954 & 10.31 & 4.9860 & 1160 \\
\hline PZU & 443598 & 0.000001 & 0.000007 & 0.3516 & 11.57 & 5.9110 & 1580 \\
\hline ANDR & 183768 & 0 & 0.07 & 3.79 & 41.07 & 43.93 & 538 \\
\hline EBS & 326368 & 0 & 0.12 & 3.74 & 23.12 & 25.71 & 506 \\
\hline OMV & 159464 & 0 & 0.08 & 4.07 & 47.54 & 37.02 & 759 \\
\hline VOE & 214768 & 0 & 0.17 & 6.87 & 35.14 & 39.42 & 846 \\
\hline
\end{tabular}


The impact of asynchronous trading on Epps effect...

The analysis of the Epps effect on both stock markets requires transformation of the tick-by-tick data into returns of different lengths. We conducted this transformation of the tick-by-tick data into a return series for the chosen intervals by the $\mathrm{R}$ code (which is available upon request from the authors).

In Figure 3 and the following figures, we present plots for the transformed data for companies from both stock markets. The most important difference between the tick-by-tick data and the transformed data is that the latter's length is fixed and the labels (times) are fixed. For an easy visual inspection, 1-minute data in Figures 3 and 4 and 10-minute data in Figures 5 and 6 are included. The return is computed as the difference between the last trades before a certain time divided by the prior one. The return in the first interval from the chosen time span is calculated by defining the prior as the first transaction of the day. If the case of a lack of transactions in the last interval, we set by definition the return at zero. It is clear that this problem takes place in cases when narrow intervals are defined. Visualized return series are similar to discrete data for an asset whose maximum and minimum prices on the chosen day do not much differ. The changes of prices themselves are discrete because of the tick size. The calculated returns are transformed ticks denominated by the current price. Hence, if the price is stable during the day, the time series of returns looks discrete.
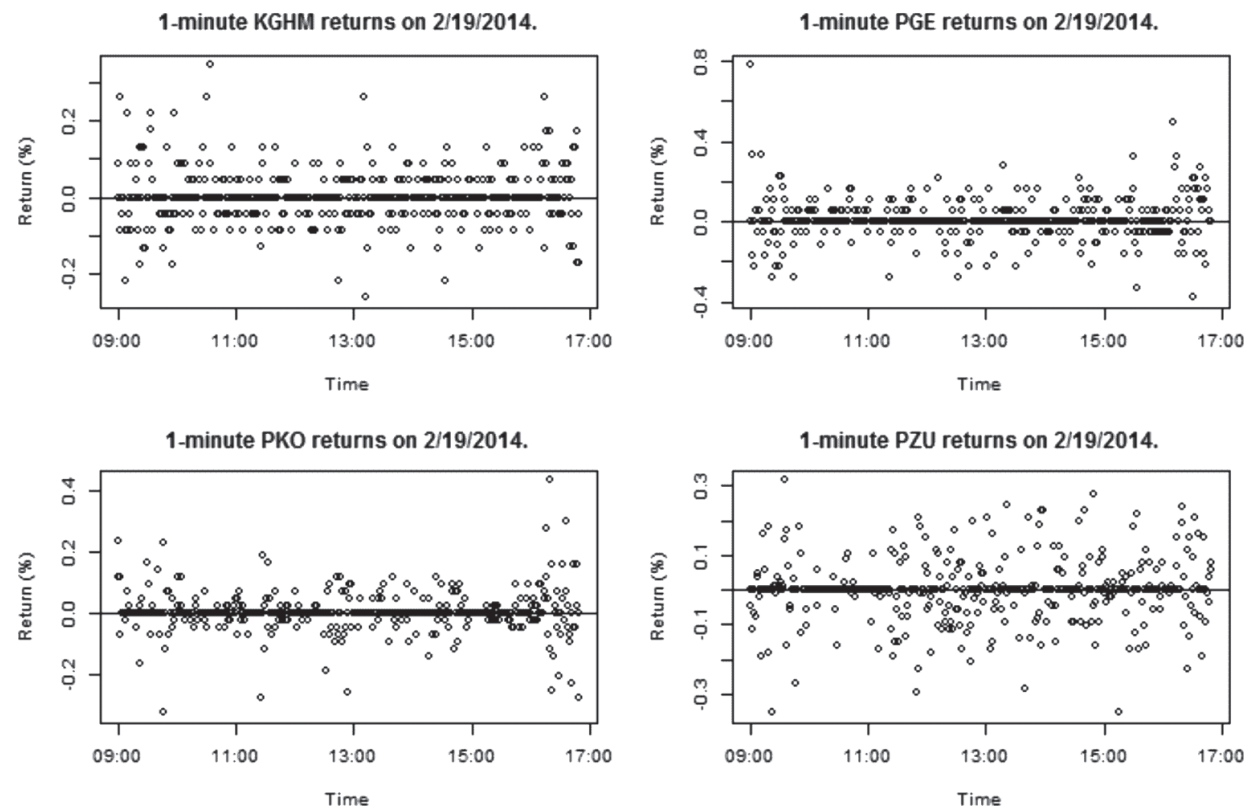

Figure 3. 1-minute percentile returns of KGHM, PKO, PGE, and PZU on February 19, 2014 

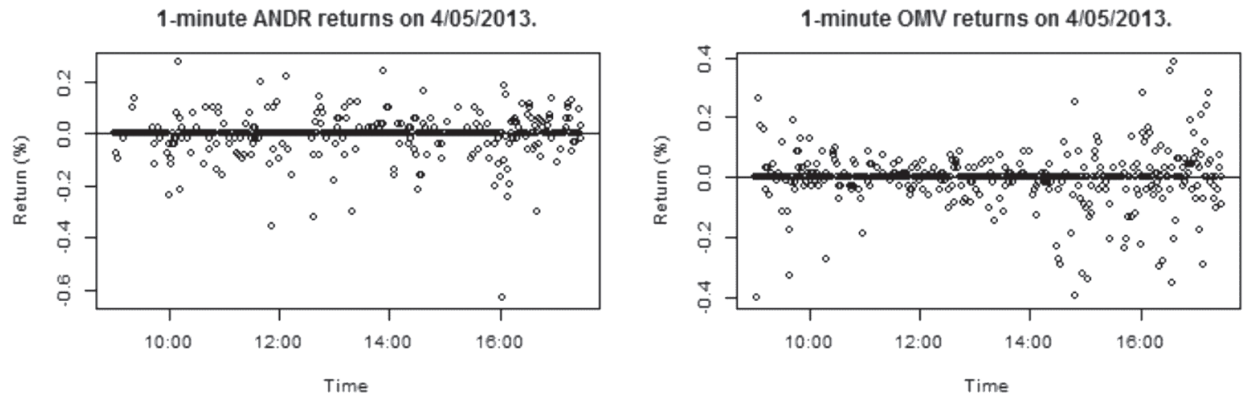

1-minute EBS returns on 4/05/2013.
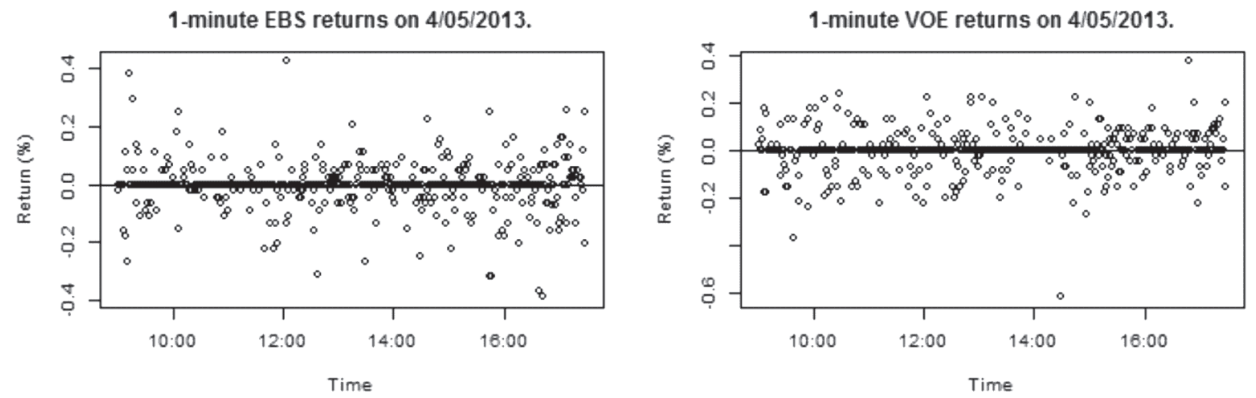

Figure 4. 1-minute percentile returns of ANDR, EBS, OMV, and VOE on April 5, 2013
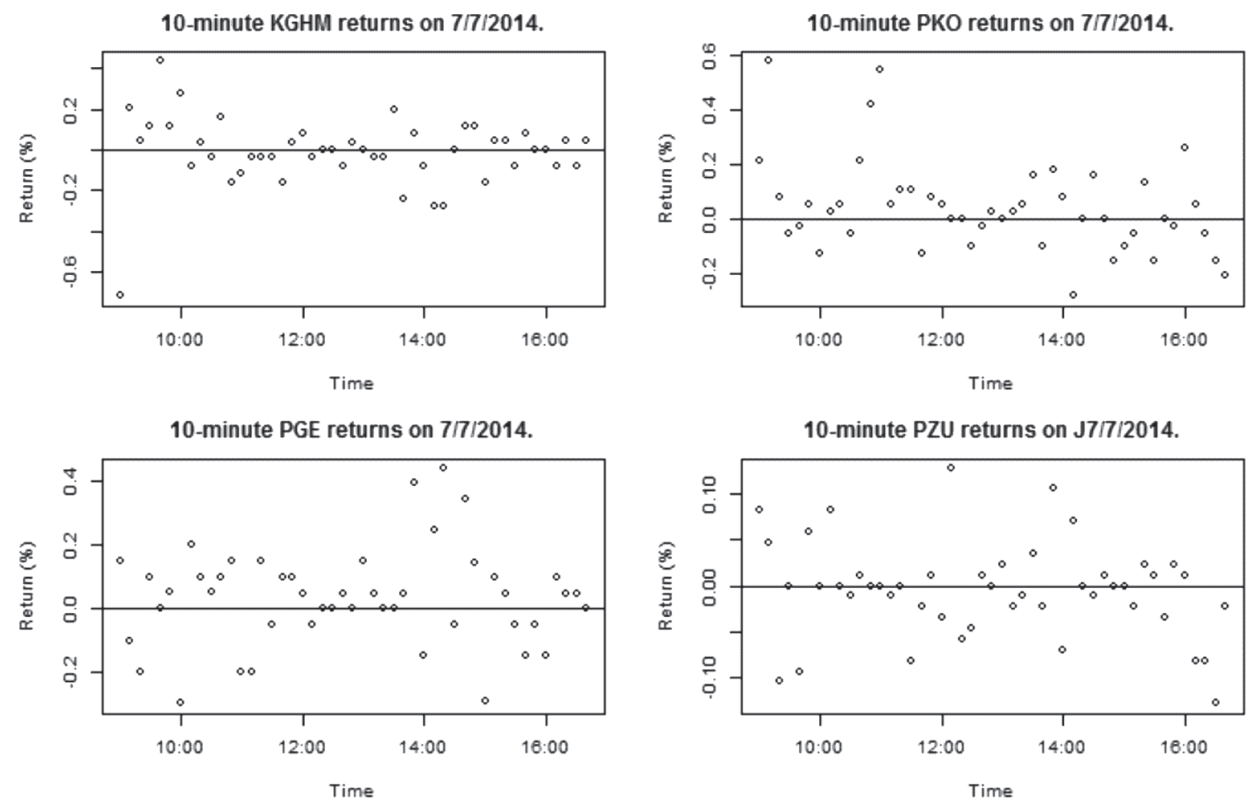

Figure 5. 10-minute percentile returns of KGHM, PKO, PGE, and PZU on July 7, 2014 
The impact of asynchronous trading on Epps effect...
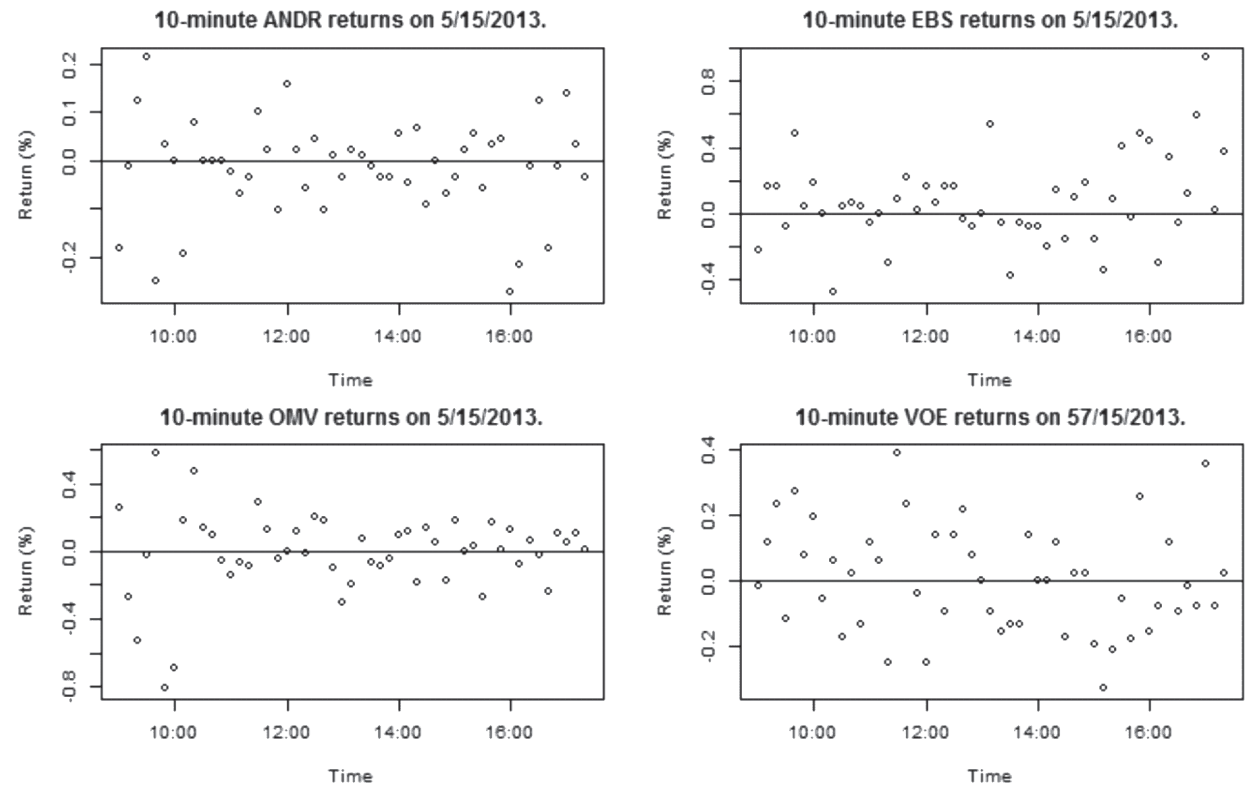

Figure 6. 10-minute percentile returns of ANDR, EBS, OMV, and VOE on May 15, 2013

It is clear that descriptive statistics of the returns may depend on the sampling frequency, stock market, stock, and particular day under study. We include descriptive statistics for 1-, 5-, and 10-minute returns of the four assets from each of the WSE and VSE. These statistics were computed independently on each day; therefore, we obtained 182 numbers in the case of the WSE and 248 numbers in the case of the VSE for each statistic and for each stock. The included boxplots illustrate the results and show variability across sampling frequencies, stocks, and days.
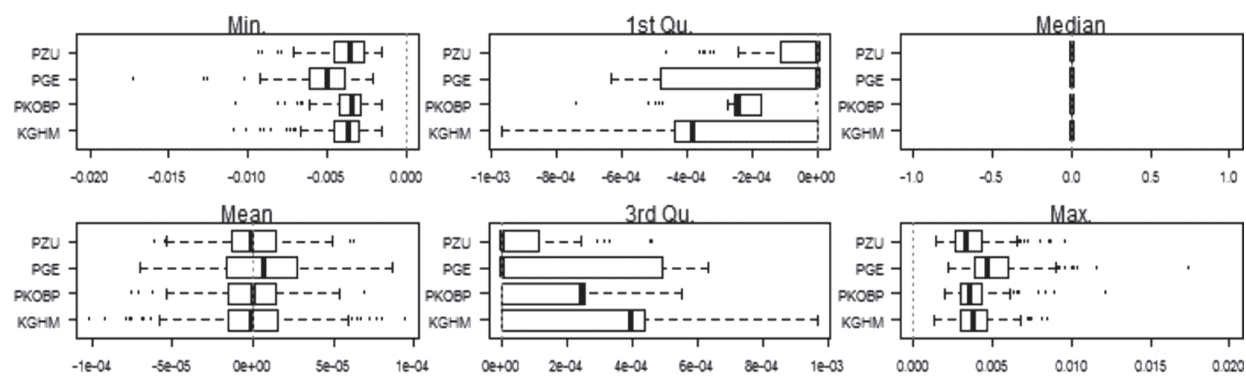

Figure 7. Descriptive statistics for 1-minute percentile returns for every trading day during 1/1/2014-9/22/2014 for WSE stocks. For minimum and maximum, the two extremely outlying observations have not been presented 
Figures 7, 8, and 9 stand for six descriptive statistics (minimum, first quartile, median, mean, third quartile, and maximum) computed for the intraday returns of each Polish stock and every day. Since the dataset includes 182 trading days, each boxplot is constructed using 182 numbers. Analogously, Figures 10, 11, and 12 are prepared for Austrian stocks.
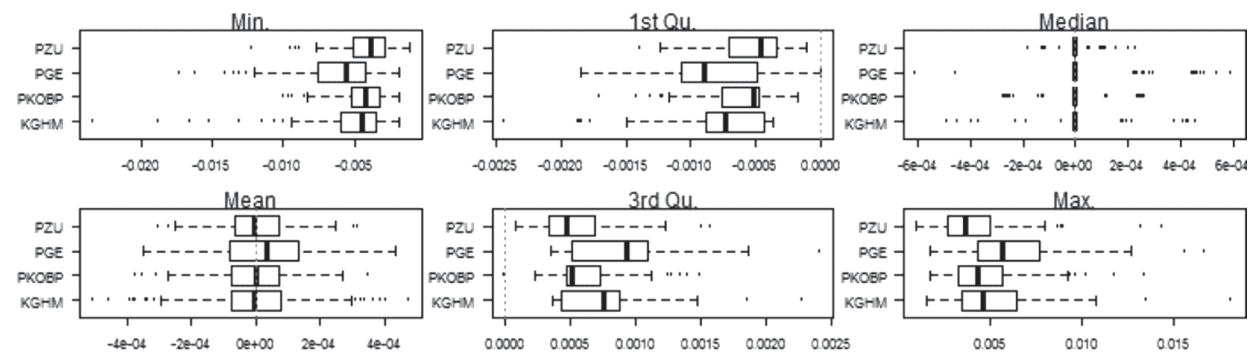

Figure 8. Descriptive statistics for 5-minute percentile returns for every trading day during 1/1/2014-9/22/2014 for WSE stocks
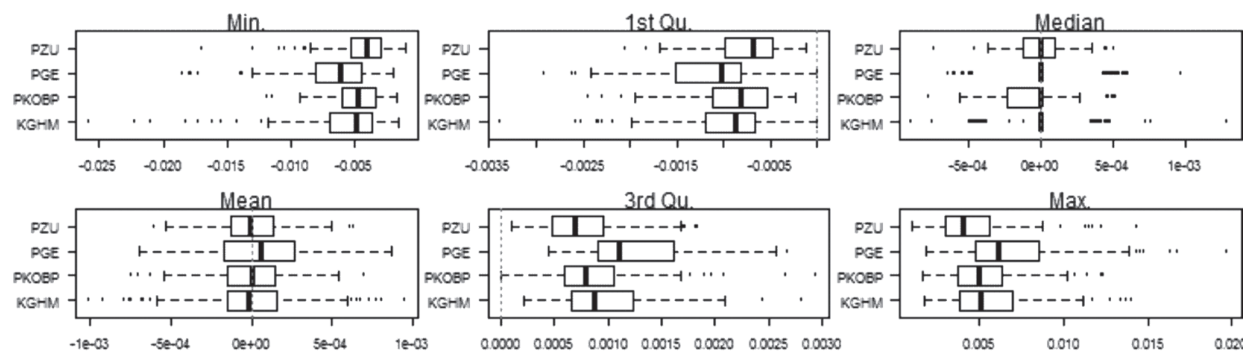

Figure 9. Descriptive statistics for 10-minute percentile returns for every trading day during 1/1/2014-9/22/2014 for WSE stocks
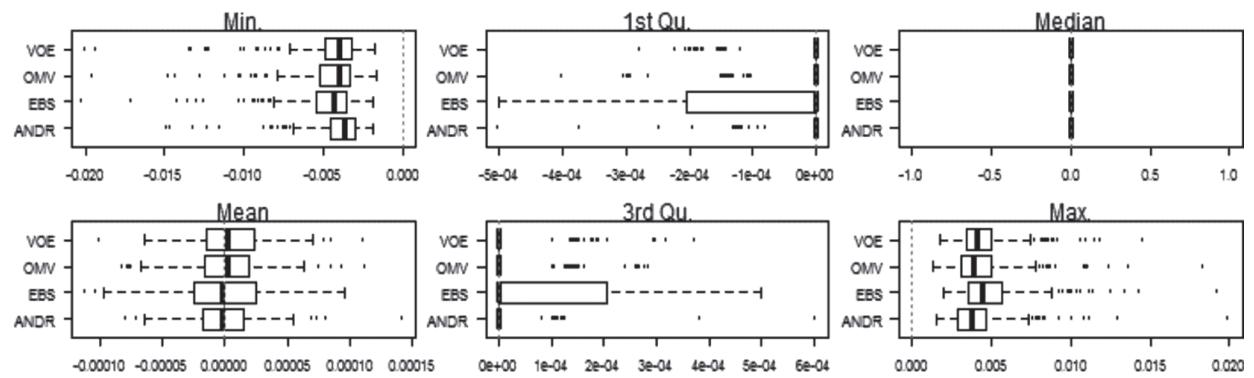

Figure 10. Descriptive statistics for 1-minute percentile returns for every trading day during $1 / 1 / 2013-12 / 31 / 2013$ for VSE stocks 
The impact of asynchronous trading on Epps effect...
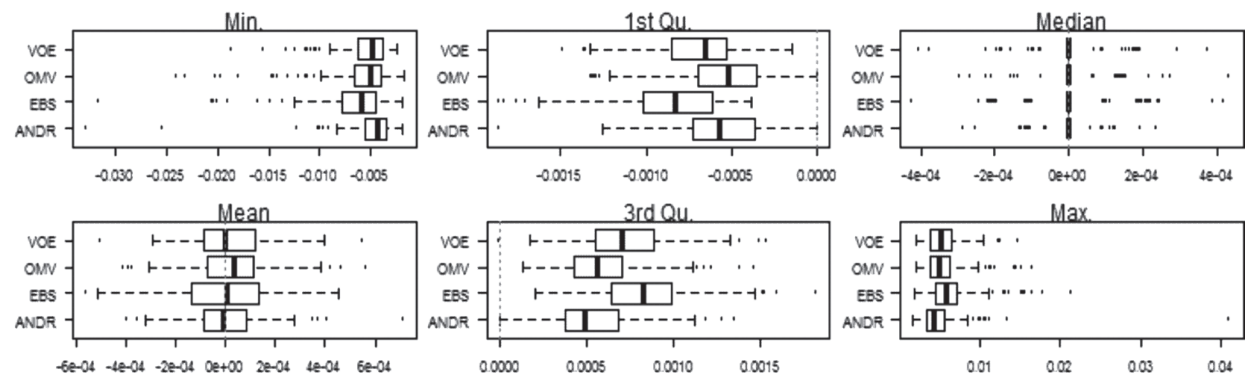

Figure 11. Descriptive statistics for 5-minute percentile returns for every trading day during $1 / 1 / 2013-12 / 31 / 2013$ for VSE stocks
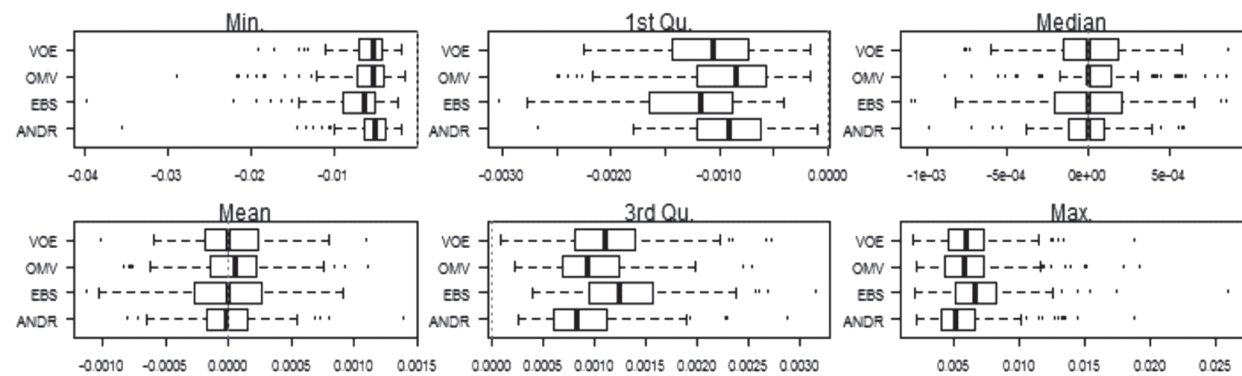

Figure 12. Descriptive statistics for 10-minute percentile returns for every trading day during $1 / 1 / 2013-12 / 31 / 2013$ for VSE stocks

\section{Epps effect}

In this part of the paper, we briefly outline the model of compensation in the correlation estimation for the asynchronous trading. Moreover, we include the visualization of the Epps effect for companies from both markets under study. In the paper, the impact of asynchronous trading times on correlation is demonstrated. As explained in an earlier section of the paper, the series of returns from intervals of a certain length is, in fact, the series of returns from intervals between the time of the last trade before the beginning of the original interval to the time of the last trade before the original interval ends. This is the reason why the observed correlation is lower than the correlation between unobserved real-price series.

The method for compensation is based on the following theorem (which is proven in Gurgul and Machno, 2015): 
Notation:

Let, for $i=1,2$, the set $\left\{r_{\Delta t}^{i}(t)\right\}_{t=0, \Delta t, \ldots, T}$ be the observed return's time series for asset $i$ on time interval length $\Delta t$; let $\left\{\tilde{r}^{i}(t)\right\}_{t=0, \Delta \tilde{t}, \ldots, T}$ be the underlying true return's time series, where $\Delta \tilde{t}$ is a unit of time. Let $\tilde{g}^{i}(t)$ and $g_{\Delta t}^{i}(t)$ be the normalized returns defined in (2) and (3); $N_{\Delta t}^{i}(t)$ is the number of $\Delta \tilde{t}$ intervals in interval $\left[\gamma^{i}(t), \gamma_{+}^{i}(t)\right]$, where $\gamma^{i}(t)$ is the last trade of asset $i$ before time $t$ :

$$
\gamma_{+}^{i}(t):=\min _{\substack{j=1,2, \ldots \\ \gamma^{i}(t+j \Delta t) \neq \gamma^{i}(t)}}\left\{\gamma^{i}(t+\mathrm{j} \Delta t)\right\}
$$

If the following assumptions hold:

A1. Series $N_{\Delta t}^{i_{1}}(t)$ are independent of each other, and of series $\tilde{r}^{i_{2}}(t)$, for $i_{1} \neq i_{2}$

A2. Variables $\tilde{r}^{1}(t)$ and $\tilde{r}^{2}\left(t+t_{1}\right)$ are independent, for $t_{1} \neq 0$

then the corrected correlation (the correlation between real unobserved series) is calculated as:

$$
\operatorname{corr}_{c}\left(r_{\Delta t}^{1}, r_{\Delta t}^{2}\right):=\operatorname{corr}\left(\tilde{g}^{1}, \tilde{g}^{2}\right)=\frac{1}{T} \sum_{j=1}^{T} g_{\Delta t}^{1}(t) g_{\Delta t}^{2}(t) \frac{\sqrt{\left\langle N_{\Delta t}^{1}\right\rangle\left\langle N_{\Delta t}^{2}\right\rangle}}{\bar{N}\left(t_{j}\right)}
$$

where $\langle\ldots\rangle$ means the average over analyzed period $[0, \mathrm{~T}]$ and $\bar{N}(t)$ is the number of $\Delta \tilde{t}$ intervals in interval $\left[\max \left\{\gamma^{1}(t), \gamma^{2}(t)\right\} ; \min \left\{\gamma^{1}(t+\Delta t), \gamma^{2}(t+\Delta t)\right\}\right]$.

In order to check the existence and properties of the Epps Effect on both stock markets under study, we transformed tick data into intraday data with intervals between 1 and 30 minutes with frequency by 1 second. It is possible to transform tick-by-tick data into intraday data of narrower intervals. However, this time series with very narrow intervals would not be more informative. Moreover, it would not be easy to conduct from the point of view of software capacity. Of course, the transformed time series is longer for narrower time intervals; for example, there are more 1-minute returns than 10-minute returns during the trading day.

The Epps effect takes place among all pairs of stocks listed on the WSE. Figures 13 and 14 show averaged correlations between the analyzed stocks and averaged corrected correlations for stocks from the WSE and VSE, respectively. The adjusted values corresponding to realized (not corrected) correlations are calculated as follows: for each trading day out of 182 days (in the case of the WSE) and 248 days (VSE) under study, we converted data into intraday data with the given interval. The length of the interval is marked on the x-coordinate of the point. In the next step, we computed correlations between returns of stocks belonging to our sample on each day. The average of these 182 correlations (or 248 , in the case of the VSE) constitutes the y-coordinate of the particular point. 
The impact of asynchronous trading on Epps effect...

The value corresponding to the corrected correlation is obtained analogously. Instead of correlations between returns, values calculated using formula (1) are averaged.

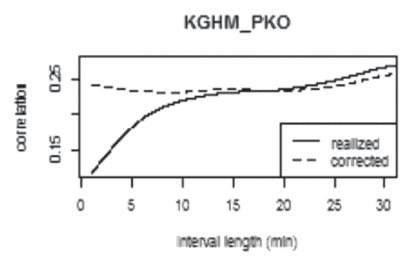

PKO_PGE

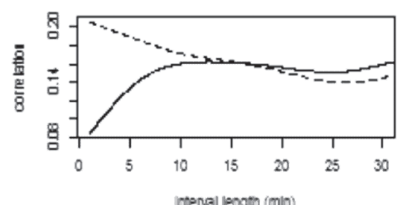

Figure 13. Realized and corrected correlations between stock returns according to data interval length for WSE stocks

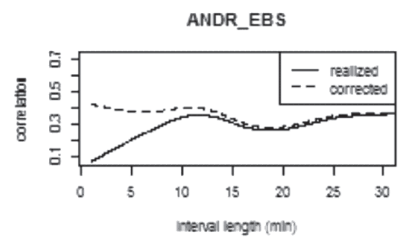

EBS_OMV

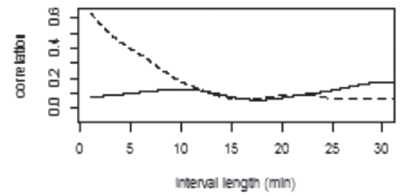

Figure 14. Realized and corrected correlations between stock returns according to data interval length for VSE stocks
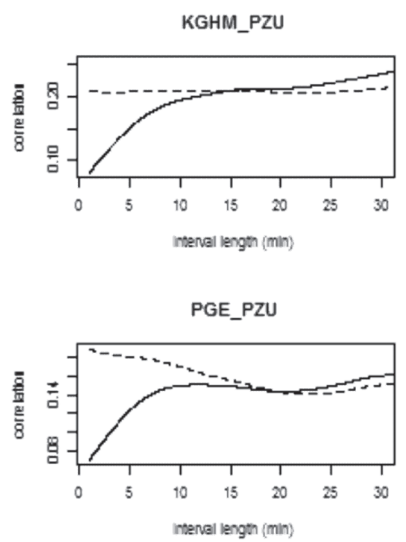

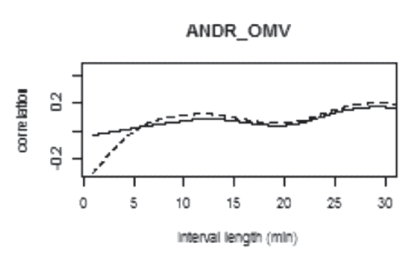

EBS_VOE

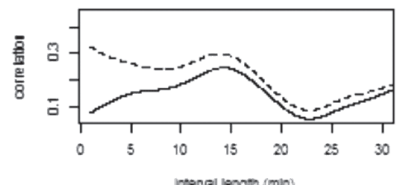

ANDR_VOE

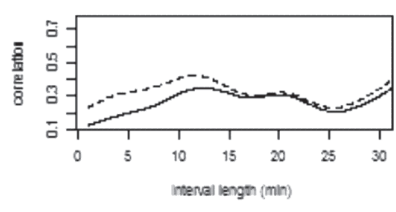

OMV_VOE

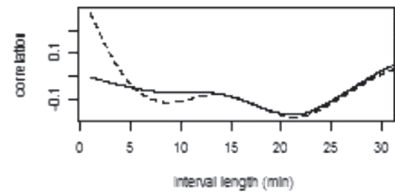

Analyzing Figures 13 and 14, we make an interesting observation. In the case of the WSE, the correction clearly works; the Epps effect is not visible after correction. However, it appears that the corrected correlations might exhibit an opposite property. The corrected correlation becomes lower for wider intervals in some cases. From a theoretical point of view, the corrected correlation should be constant under the assumptions of the presented theorem. It is true that the assumptions are 
not fully met; however, one could expect that, even after the correction, a smaller Epps effect caused by other factors (such as lead-lag correlation or the discreteness of the data) is still present. Another surprising observation is the lower value of corrected correlation than the realized correlation for wider intervals.

The observed correlation and corrected correlation for the VSE is much more surprising, especially as compared to the corresponding results for the WSE. The correlations between Austrian stocks do not possess a typical pattern. We observe that the VOE and the OMV are basically uncorrelated on the intraday level; thus, the analysis does not make much sense. The ANDR and EBS constitute the most-correlated pair. We observe that, in the case of this pair, the patterns of the correlation and the corrected correlation are similar to pairs from the WSE.

\section{Conclusions}

The correlations between high-frequency financial data are strongly affected by asynchronous trading. Contrary to widespread beliefs, our study suggests that asynchronous trading may be the only essential cause of the Epps effect in the data under study on the WSE. However, the presence of the Epps effect on the VSE is not clearly pronounced. The Polish stocks under study are traded on one stock exchange (no dual listing) and are among the most-liquid assets in Warsaw. In correlations with the asynchronous effect removed, the Epps effect is no more visible.

The analysis does not show uniform properties among Austrian assets. For the most-liquid and most-correlated pair (namely, ANDR-EBS), the analysis shows similar results as for Polish stocks. However, for less-liquid and less-correlated assets, the correlation pattern itself does not exhibit a clear Epps effect. Moreover, the suggested correction seems to not work, especially for the ANDR-OMV pair.

The hypothesis that there are others significant sources of the Epps effect on the WSE seems to be false. However, on the VSE, the existence and sources of the Epps effect is not clear; therefore, additional analysis is needed.

In addition (in the case of the WSE), the relationship between the corrected correlation and sampling frequency shows an unexpected pattern. First of all, the corrected correlation is lower for wide intervals (wider than 20-minute) for all pairs from the sample. The second unexpected observation is that, in some corrected correlation patterns, evidence for the reciprocal Epps effect was found (i.e., the corrected correlation decreases as the sampling frequency of data decreases).

In the case of the VSE, the correlation does not increase when the sampling frequency decreases in some pairs and intervals (indicating a lack of the Epps effect). There is no visible pattern for the corrected correlations; for instance, the 
correction seems to overestimate the correlation for the OMV-VOE pair for highfrequency data (left side of the graph in Figure 14). However, for the ANDR-OMV pair, the correction seems to underestimate the correlation. Moreover, corrected correlations are not lower than observed correlations for less-frequent data, which is the case on the WSE.

The main question in the context of this analysis is the connection between the intensity of trading and the Epps effect. From a theoretical point of view, the impact of asynchronous trading on the Epps effect should be stronger for lessliquid assets. However, the conjecture that the Epps effect is stronger for less-liquid pairs (the Austrian assets are less liquid than Polish ones) is not confirmed in this study. The addressed topics need further research based on the recent data.

\section{References}

[1] Conlon, T., Ruskin, H.J. and Crane, M. (2009) 'Multiscaled Cross-Correlation Dynamics In Financial Time-Series, Physica A 388(5), pp. 705-714.

[2] Epps, T.W. (1979) 'Comovements in stock prices in the very short run',Journal of the American Statistical Association, vol. 74, pp. 291-298.

[3] Gurgul, H. and Machno, A. (2015) 'The Impact of Asynchronous Trading on Epps Effect on Warsaw Stock Exchange', Central European Journal of Operational Research, forthcoming, DOI: 10.1007/s10100-016-0442-y.

[4] Lundin, M., Dacorogna, M. and Muller, U.A. (1998) 'Correlation of High Frequency Financial Time Series', in Lequeux P. (ed.) Financial Markets Tick by Tick, Wilney and Sons.

[5] Mastromatteo, I., Marsili, M. and Zoi, P. (2011) 'Financial correlations at ultrahigh frequency: theoretical models and empirical estimation', The European Physical Journal B, vol. 80, pp. 243-253.

[6] Munnix, M.C., Schafer, R. and Guhr, T. (2010) 'Compensating asynchrony effects in the calculation of financial correlations', Physica A 389, pp. 767-779.

[7] Toth, B. and Kertesz, J. (2009) 'The Epps effect revisited', Quantitative Finance, vol. 9, pp. 793-802. 